\title{
THE USE OF AUTOMATED "ON LINE" GASCHROMATROGRAPHY FOR THE MONITORING OF ORGANIC TRACE GASES IN THE ATMOSPHERE AT LOW LEVELS
}

\author{
J. RUDOLPH, F. J. JOHNEN, A. KHEDIM AND G. PILWAT* \\ Institut für Atmosphärische Chemie, Kernforschungsanlage Jülich GmbH, Postfach \\ 1913, D-5170 Jülich, FRG
}

The usual procedure for measurements of hydrocarbons, halocarbons, and similar trace gases in the atmosphere is sample collection in the field and subsequent analysis in the laboratory. However this procedure is not adequate if intensive field investigtions with a high measuring frequency are desired. In this case the use of in-situ measurement techniques is necessary. For the measurement of volatile organic trace substances in the atmosphere the by far best technique is gaschromatography. However even if very sensitive ionization detection is employed, a preconcentration step is necessary if the lechnique is to be used at low pollution levels. In this paper the basic design and some applications are described for an automated gaschromatograph suitable for unattended operation. The instrument allows automated prconcentration of the ambient air sample, injection etc. including calibration with an external standard. The lower limits of detection are in the ppt range, the reproducibility of the measurements better than $10^{\circ}$. Some examples for results obtained with this instrument are presented.

KEY WORDS: Atmospheric pollution, organic trace gases. gaschromatography, sample preconcentration, automation

\section{INTRODUCTION}

The concern for the problem of atmospheric pollution has increased strongly during the past years. It is by now generally accepted, that man made atmospheric trace constituents can severely affect our environment not only on a local or regional scale but even on a global basis. The number of different atmospheric trace compounds relevant for air quality and regional or global pollution is considerable. Furthermore it has been recognized that emissions from the biosphere play an important role in the chemistry of our atmosphere. With few exceptions the mixing ratios of organic trace compounds in the atmosphere are in the ppb and ppt range. Many of these compounds exhibit large variations not only in space, but also in time (e.g. diurnal or seasonal cycles). Often concentration changes by an order of magnitude can be observed within a few hours. In order to understand the impact of the various trace compounds in the atmosphere, we have

*Institut für Chemie der Belasteten Atmosphäre, Kernforschungsanlage Jülich GmbH. Postfach 1913, D.5170 Jülich, FRG. 
to know and understand their atmospheric abundance as a function of time and space.

\section{GENERAL CONSIDERATIONS}

The nowadays most widely used method for measurements of organic trace constituents in the atmosphere is gaschromatography. ${ }^{1.2 .3}$ Generally the air samples are collected either as whole air samples or by preconcentration of the organic species on an adsorbent. The collected samples are subsequently analyzed in the laboratory. ${ }^{1.2 .4 .5 .6}$ The advantages of this procedure are: the amount of instrumentation in the field is minimized and the analysis of the samples can be made in the laboratory under optimum conditions. However, if frequent measurements are needed, sample collection and subsequent transfer into the laboratory can be rather time consuming. Furthermore, during sample collection and transport sample degradation or contamination can occur. The necessary technical effort to ensure sample integrity is rather high and often not easily maintained for routine measurements if a high sample throughput is necessary. Evidently the better solution is to place the instrument in the field and measure "in situ". For continuous measurements over longer periods only automated measurement techniques are sufficiently economical.

In this paper we will describe techniques which we successfully used for in situ measurements and, which were fully automated including an automated preconcentration and which require attention by an operator once a day or less frequently.

\section{RESULTS AND DISCUSSION}

\section{Experimental}

In principle, the experimental techniques we applied were similar to those described previously. ${ }^{2.7 .9}$ The gaschromatographs we used are Sichromat I or II (Siemens, Karlsruhe, FRG) with flame ionization, FID, and electron-capture detectors, ECD. We also used photo ionization detectors (hnu, Massachusetts, USA) in combination with FID and ECD. All carrier gases were of 5.0 quality (impurities less than $10^{-5}$ ) or better and were further purified by passing them through an absorber packed with a mixture of molecular sieve (5 and 10 A) and active charcoal (GC-grade, Merck, Darmstadt, FRG).

The samples were injected by means of multiport valves (Valco, Switzerland), the complete inlet systems were constructed and built at our institute with metal bellows valves (Nupro-Company, Ohio, USA) and Valco-multiport valves. The valves were actuated pneumatically, the solenoid valves which switch the pressurized gas are operated by the GCs which are microprocessor controlled. 


\section{Preconcentration}

As already mentioned, for measurements outside urban or industrialized areas, a preconcentration step has to be used prior to the analysis if adequate lower limits of detection are wanted. The sample volumes range between a few hundred and several thousand $\mathrm{cm}^{3}$ (STP).'1.2.5.7.8

A trapping procedure which is used quite frequently for a number of different low and medium molecular weight organic trace gases is adsorption on a short column, in general between 2 and $4 \mathrm{~mm}$ i.d., about $15-25 \mathrm{~cm}$ long packed with porous glass beads. ${ }^{2.3 .7 .9}$ For efficient trapping of light trace gases porous glass beads have to be cooled with liquid air, argon or nitrogen. Air is drawn through the cooled adsorption tube which retains nearly all atmospheric components except nitrogen and oxygen. The advantage of porous glass beads is, that organic trace compounds with boiling points up to $420 \mathrm{~K}$ can be desorbed quantitatively at temperatures below $350 \mathrm{~K}^{2}$. This allows fast desorption of the preconcentrated substances at temperatures where thermal decomposition is negligible. Also contamination and memory effects are avoided.

Furthermore porous glass beads are not degraded by reactive atmospheric trace constituents such as ozone, hydrogenperoxide, nitrogen dioxide etc. Thus the stability of the adsorbent presents no problem, even for long term continuous measurements. The main difficulty is to reduce the consumption of the cryogenic liquid (e.g. liquid $\mathrm{N}_{2}$ or $\mathrm{Ar}$ ) to a level that is acceptable for field operation. The most obvious way for an automated cooling system is to use a dewar filled with liquid nitrogen which is moved mechanically up and down (Figure 1). For preconcentration the dewar is moved upward by the motor driven cog wheel until the upper liquid $\mathrm{N}_{2}$ sensor is immersed in the cryogenic medium. After preconcentration. the dewar is moved downwards until the lower sensor is above the liquid nitrogen surface and the sample loop can be heated for desorption of the trapped trace compounds. The two liquid $\mathrm{N}_{2}$ sensors make sure, that the sample loop always has a defined position relative to the liquid $\mathrm{N}_{2}$ level. Thus a reproducible preconcentration is obtained, even if the liquid nitrogen level in the dewar changes. With a dewar of $3 \mathrm{dm}^{3}$ volume $(70 \mathrm{~cm}$ high, about $10 \mathrm{~cm}$ i.d.) such a system can operate more than $24 \mathrm{~h}$ at a rate of one sample of $2 \mathrm{dm}^{3}$ (STP) every $2 \mathrm{~h}$ before liquid nitrogen has to be refilled. For longer periods of unattended operation this technique is less suitable. Either the size of the dewar vessel has to be increased or the instrument has to be equipped with an automated refilling system from a larger storage tank.

An alternative for cryogenic preconcentration which avoids essentially all moving parts and thus reduces the risk of instrument failure is described in Figure 2. Instead of moving the complete liquid nitrogen reservoir, only the level of the cryogenic liquid is varied. The inner compartment which contains the sample loop is airtight and has an opening at the bottom. The complete vessel is immersed in liquid nitrogen. As long as the solenoid valve is open, the preconcentration loop is in contact with the cryogenic liquid. If the valve is closed, the level of liquid nitrogen is pushed downwards to the lower end of the vessel by the pressure of evaporating liquid nitrogen. The preconcentration loop can be heated for desorp- 


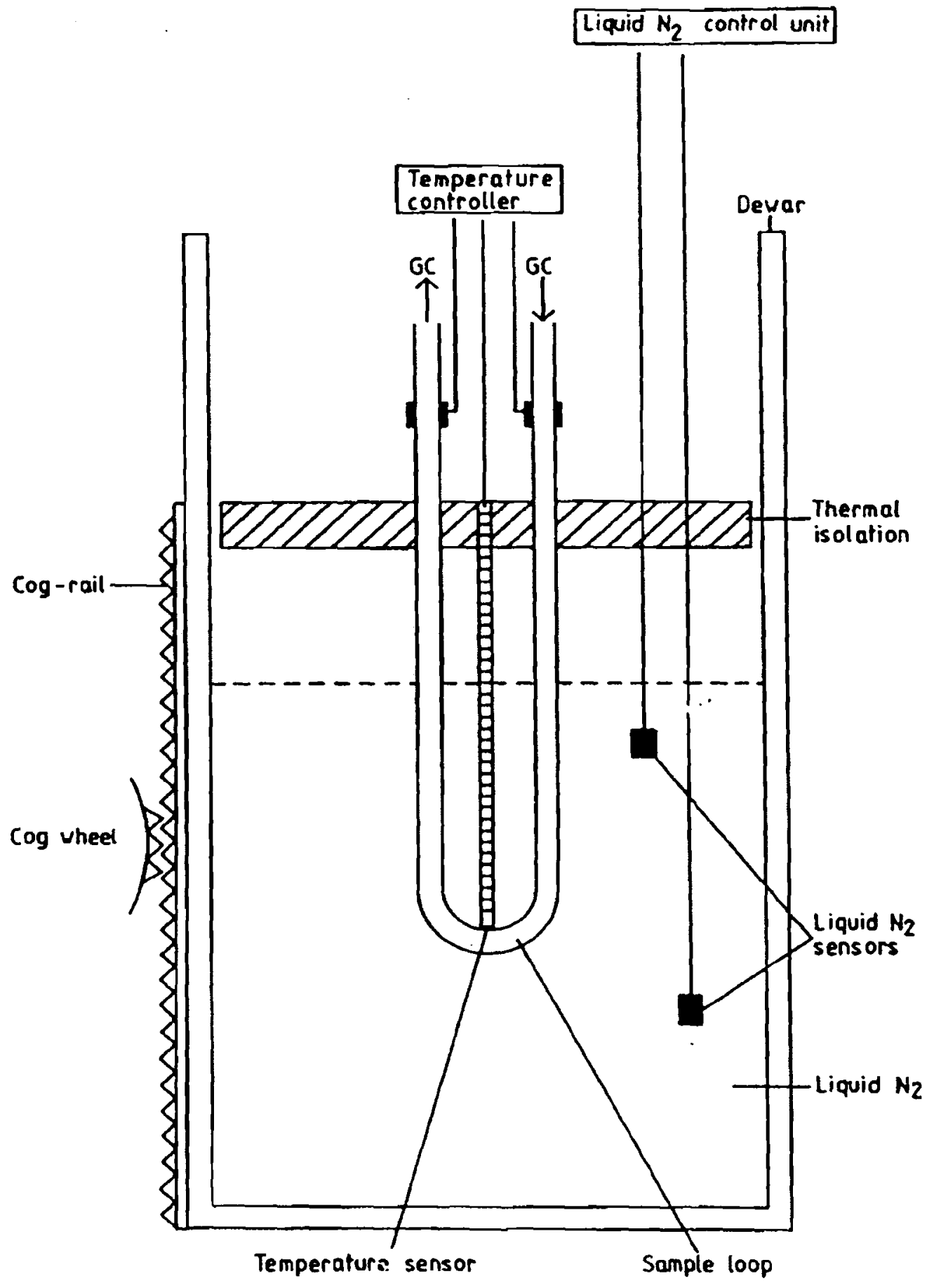

Figure 1 Schematic drawing of a cryogenic preconcentration device with a "moving dewar", details see text. 


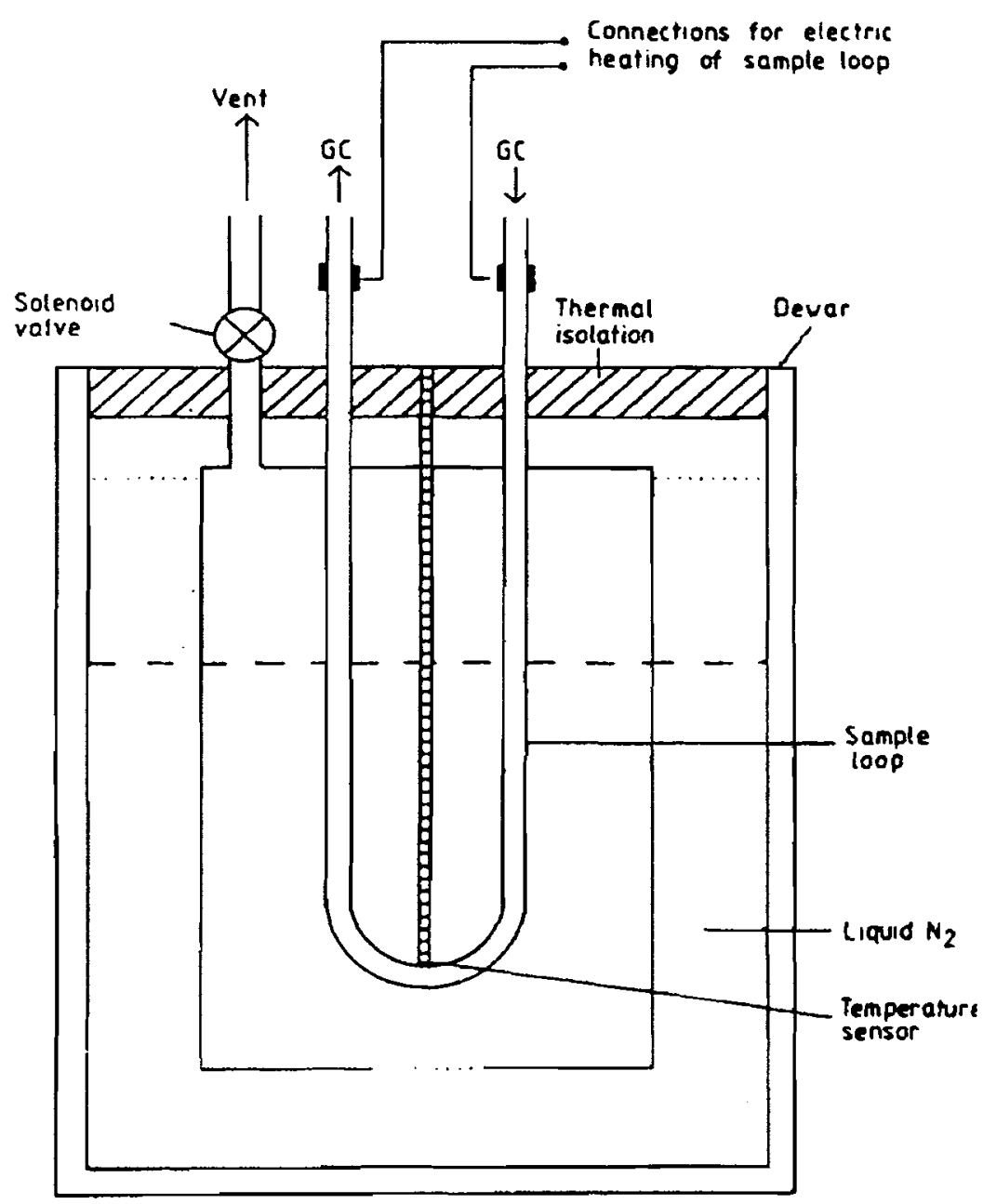

Figure 2 Schematic drawing of a cryogenic prconcentration device with "liquid nitrogen displacement", details see text.

tion of the enriched sample. The amount of liquid nitrogen which is consumed by this method of preconcentration is essentially limited to the evaporation loss of the dewar which contains the liquid nitrogen reservoir. Since the dewar is not moved, it is possible to use a high quality dewar of a large volume which has a low evaporation rate. A $20 \mathrm{dm}^{3}$ liquid nitrogen reservoir is sufficient to allow sample preconcentration for more than a week at a rate of more than 10 samples of $1-2 \mathrm{dm}^{3}$ volume (STP) per day. Furthermore, the consumption of liquid nitrogen is so low, that the evaporating liquid nitrogen can be recondensed by a commercial two stage Joule-Thompson cooler. This enables to operate such a preconcentration instrument under conditions where the supply with liquid nitrogen is not possible.

\section{Separation}

The use of multiport valves allows a controlled and reproducible injection of the enriched trace compounds. ${ }^{2.7 .9}$ The critical problem is the selection of a column which is suitable for the separation of the preconcentrated air sample. Such a 
sample contains several $\mathrm{mg}$ of water and several tenths of a $\mathrm{mg}$ of carbondioxide. Several authors describe the use of drying agents such a magnesiumperchlorate or potassiumcarbonate to remove water from the sample.1,7.10 However chemical drying agents have to be replaced or reconditioned regularly which increases the amount of maintenance. "Perma pure" permeation dryers are often used to dry moist gases. However, we observed that air samples passed through a "Nafion" tube (essentially the main part of a "Perma pure" type drying system) showed significant but unreproducible changes in the concentration of several organic trace gases. Both. losses, probably due to wall adsorption, and contamination could be observed. We have made no efforts to clean or precondition such a drying device and can therefore not draw any final conclusions on the possibility to use a "Perma pure" type gas dryer. Instead we decided not to use such a system since these few test convinced us that these types of permeation dryers are not suitable for measurements of many organic trace constituents at the sub-ppb level. Another possibility is preseparation of water and $\mathrm{CO}_{2}$ from the enriched trace gases on a packed column and refocussing of the trace substances for separation on a capillary column. ${ }^{9}$ This technique is very versatile and can be adapted for measurements of a large variety of different trace gases. However this method requires not only an additional cryotrapping step but also the use of two column ovens and was therefore also rejected. Instead we pursued methods which allow direct interfacing of the preconcentration step with the gaschromatographic separation.

The most simple way is to use a packed column which is suitable for the separation of samples containing substantial amounts of water. We combined a cryogenic preconcentration step with a separation on a $3 \mathrm{~m}$ column ( $2 \mathrm{~mm}$ i.d.) packed with Porapak QS (100-120 mesh, Waters, Massachusetts, USA). An example for the measurement of an air sample is shown in Figure 3. This combination allows the measurement of most $C_{2}-C_{6}$ hydrocarbons, several aldehydes and ketones and of some light halocarbons with detection limits of several ppt and a reproducibility of roughly $10 \%$. The disadvantage is both the rather long time necessary for the separation and the limitation to trace gases of relatively low molecular weight.

Wide bore capillary columns are often used as substitutes for packed columns if fast separations are wanted. In figure 4 an example for the separation of a preconcentrated air sample on a SE 54 wide bore capillary $(25 \mathrm{~m}, 0.52 \mathrm{~mm}$ i.d., Macherey und Nagel, Düren, FRG) is shown. Obviously the quality of the separation is not too good, a result of the rather large volume of water and carbondioxide. In combination with a detector of some selectivity (e.g. electron capture detection or photo ionization detection) a sufficiently reliable peak identification is still possible. We used such a column combined with a photo ionization detector in parallel to a packed column by splitting the preconcentrated sample between the packed column and the wide bore capillary with a ratio of $4: 1$. This combination was used to measure the most important atmospheric organic trace gases in the $C_{2}-C_{8}$ range during a six week measuring campaign on a remote mountain station in the Black Forest with a frequency of one measurement every $2.5 \mathrm{~h}$. 


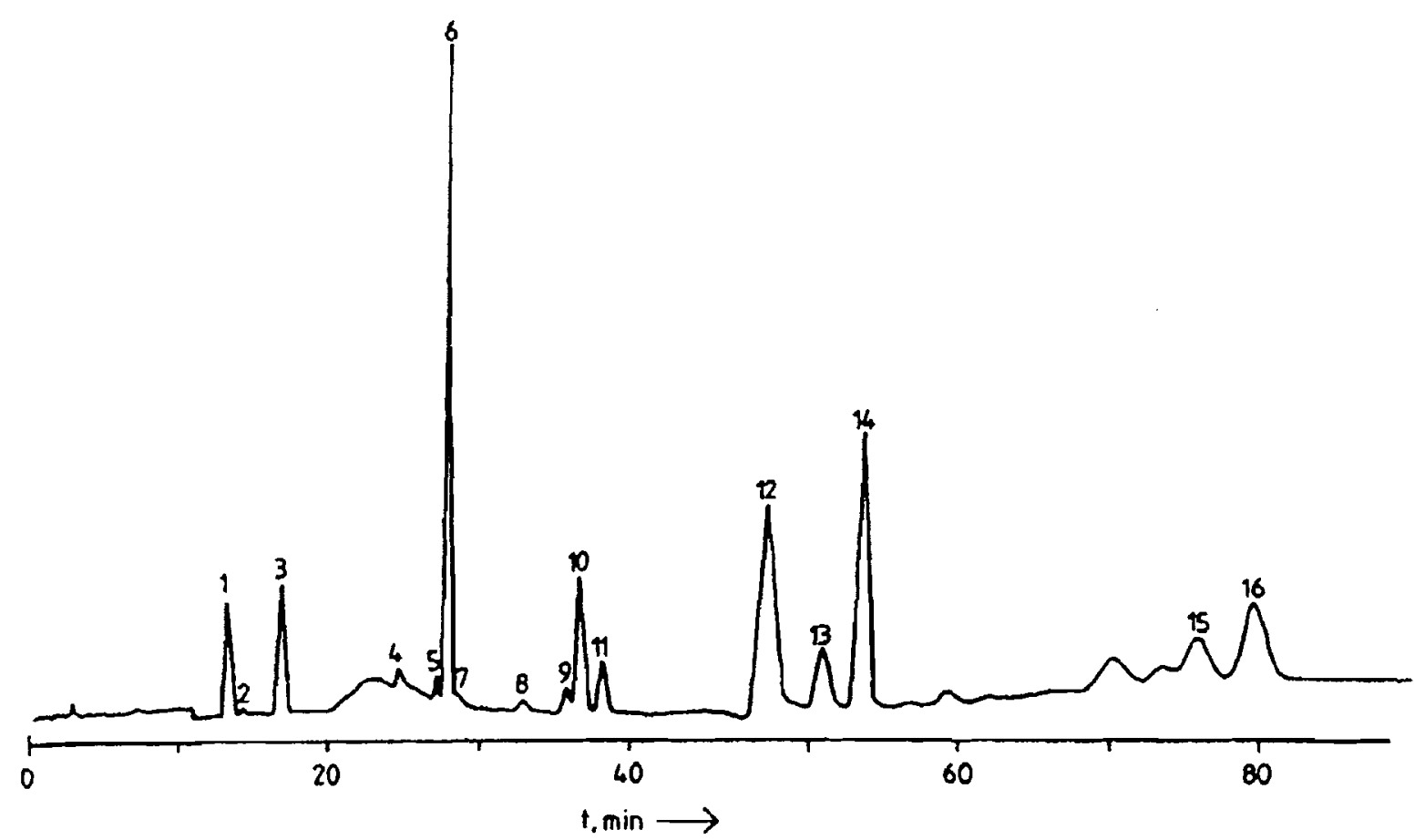

Figure 3 Separation of atmospheric trace gases on a packed column (Porapak QS, 100-120 mesh, $3 \mathrm{~m}$ leng(h, $2 \mathrm{~mm}$ i.d.), sample volume $1.5 \mathrm{dm}^{3}$ (STP). Temperature program: $20 \mathrm{~min}$ at $290 \mathrm{~K}$, $5 \mathrm{~min}$ with $20 \mathrm{Kmin}^{-1}, 20 \mathrm{~min}$ at $2 \mathrm{~K} \mathrm{~min}^{-1}$ then with $0.5 \mathrm{Kmin}^{-1}$ to $350 \mathrm{~K}$. Detector: FID, Carrier gas $\mathrm{N}_{2}$, $30 \mathrm{~cm}^{3} \mathrm{~min}^{-1}$. 1: Ethene, 2: Acetylene, 3: Ethane, 4: Water, 5: Propene, 6: Propane, 7: Methylchloride, 8: Acetaldehyde, 9: i-Butane, 10: 1-Butene, 11: n-Butane, 12: Acetone, 13: i-Pentane, 14: n-Pentane, 15: Benzene, 16: Trichloroethene.

In the past few years wide bore porous layer open tubular (PLOT) columns with porous polymers as stationary phase have become available. These columns are more suitable for the separation of samples containing substantial amounts of water than wall coated wide bore capillary columns. Still, the capacity of these columns for water is considerably lower than for packed columns with a similar stationary phase. We can reduce the amount of water which is injected by keeping the temperature of the sample loop below $350 \mathrm{~K}$. Under these conditions, a considerable part of the water in the sample remains as a liquid in the sample loop. In a previous paper ${ }^{2}$ we have shown that under these conditions quantitative injection of organic compounds up to $\mathrm{C}_{8}$ is possible. However hydrogen has to be used as carrier gas to allow relatively high gas flow rates (about $10-15 \mathrm{~cm}^{3} \mathrm{~min}^{-1}$ ) without substantial loss of separation efficiency. Lower carrier gas flow rates cause peak broadening and tailing during sample injection. In figure 5 an example for the separation of an air sample on a $30 \mathrm{mGS}-\mathrm{Q}$ column (PLOT column of $0.53 \mathrm{~mm}$ i.d., J\&W-Scientific, California, USA) is shown.

\section{Detection}

For gaschromatographic measurements of organic trace constituents in the non urban atmosphere, detectors are needed which enable identification and quantifi- 


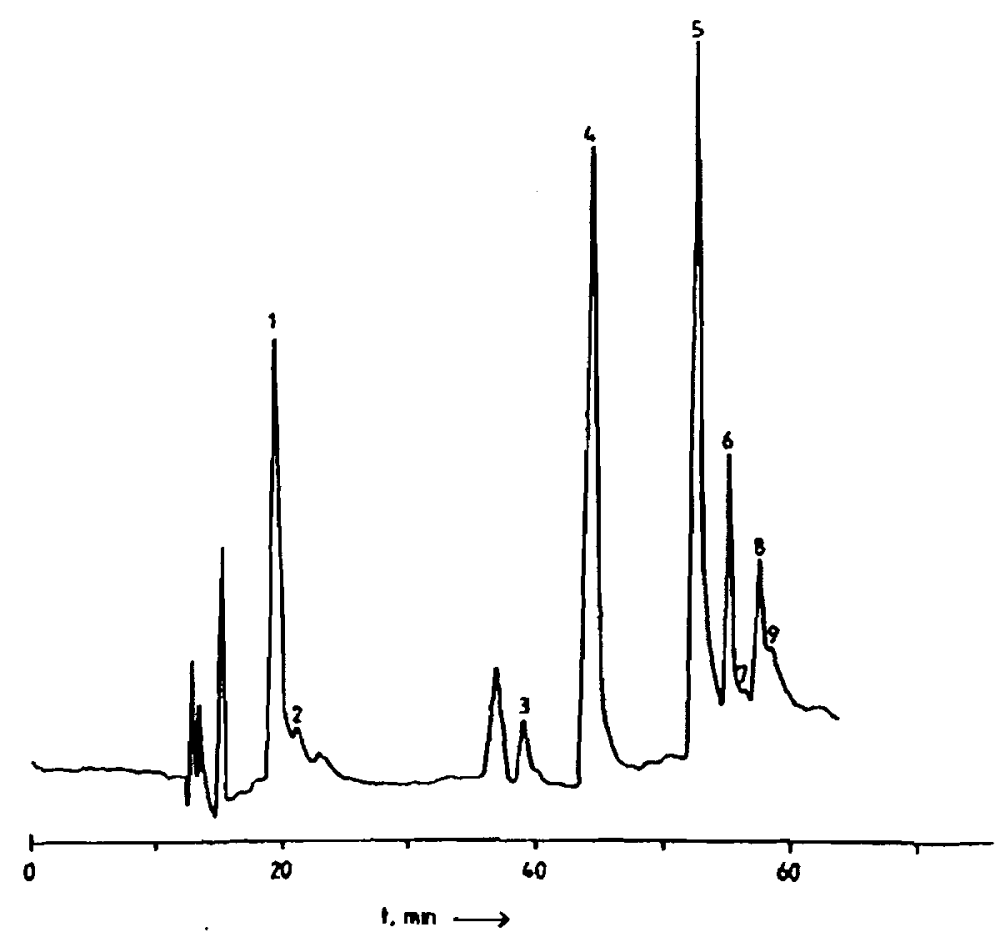

Figure 4 Separation of atmospheric trace gases on a wide bore capillary ( $25 \mathrm{~m}$, SE 54), sample volume $0.2 \mathrm{dm}^{3}$ (STP). Same temperature program as Fig. 3, Detector: PID, Carrier gas $\mathrm{N}_{2}, 6 \mathrm{~cm}^{3} \min ^{-1}$. 1 : Acetone, 2: Isoprene, 3: Benzene, 4: Trichloroethene, 5: Toluene, 6: Tetrachloroethene, 7: Ethylbenzene,

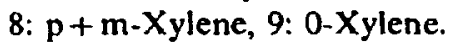

cation of peaks containing less than a nanogram of substance. Less sensitive detectors would either require a preconcentation from very large volumes of air or not allow measurements at the low concentration levels which are often found outside urban areas. Commerical ionization detectors are in general sufficiently sensitive and reliable for operation at remote stations. Most widely used are flame ionization detectors and electron capture detectors, but also photo ionization detection has been successfully used for atmospheric measurements. For certain applications also combinations of different detectors have been used. ${ }^{1,2,7,8.9}$ This has been shown to improve the number of substances which can be detected at low levels and the linear range of the measurements. It also allows better peak identification and recognition of interferences. For automated in situ measurements we generally used flame ionization detection, sometimes in combination with electron capture - or photo ionization detection. With flame jonization detection we obtained lower limits of detection of a few ppt (for a $1000 \mathrm{~cm}^{3}$ air sample) and a reproducibility better than $10 \%$. The linear range is better than $10^{5}$. With electron capture detection even better detection limits (less than one ppt) were obtained for most halogenated organic trace gases. However the linear range with electron capture detection is only about one thousand. Photo ionization detection is especially suitable for alkenes, aromatic hydrocarbons, aldehydes and ketones. Detection limits of less than one ppt can be achieved for aromatic hydrocarbons and the linear range covers more than five orders of magnitude. However the 


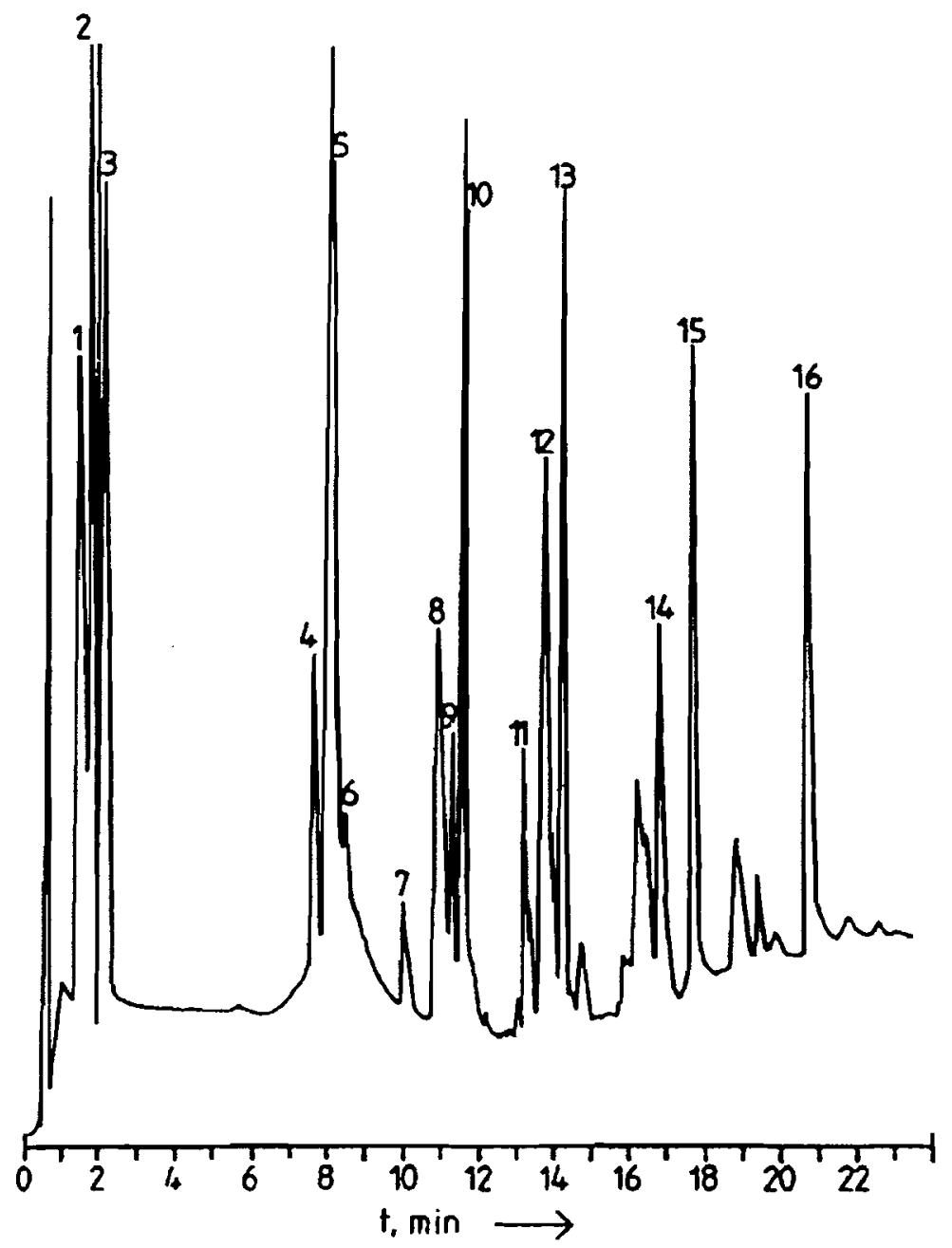

Figure 5 Separation of an air sample on a $30 \mathrm{~m} \mathrm{GS-Q} \mathrm{wide} \mathrm{bore} \mathrm{capillary,} \mathrm{sample} \mathrm{volume} 0.7 \mathrm{dm}^{3}$ (STP). Temperature program: $5 \mathrm{~min}$ at $300 \mathrm{~K}$, then $25 \mathrm{kmin}^{-1}$ for $4 \mathrm{~min}$, then with $10 \mathrm{~K} \mathrm{~min}^{-1}$ to S10 K. Detector: FID, Carrier gas $\mathrm{H}_{2}, 15 \mathrm{~cm}^{3} \mathrm{~min}^{-1}$. 1: Ethene, 2: Acetylene, 3: Ethane, 4: Propene, 5: Propane, 6: Methylchloride, 7: Acetaldehyde, 8: i-Butane, 9: 1-Butene, 10: n-Butane, 11: Acetone, 12: i-Pentane, 13: n-Pentane, 14: n-Hexane, 15: Benzene, 16: Toluene.

long-term stability of photo ionization detection is not as good as for flame ionization detection.

We quantified our field measurements by comparison with air samples of known concentrations which are kept in pressurized cylinders. These standards are measured by the same procedure as the outside air. The standards are calibrated in the laboratory by comparison with prepared mixtures of the individual substances.

\section{EXAMPLES FOR FIELD MEASUREMENTS}

During several measuring campaigns we successfully used fully automated methods 


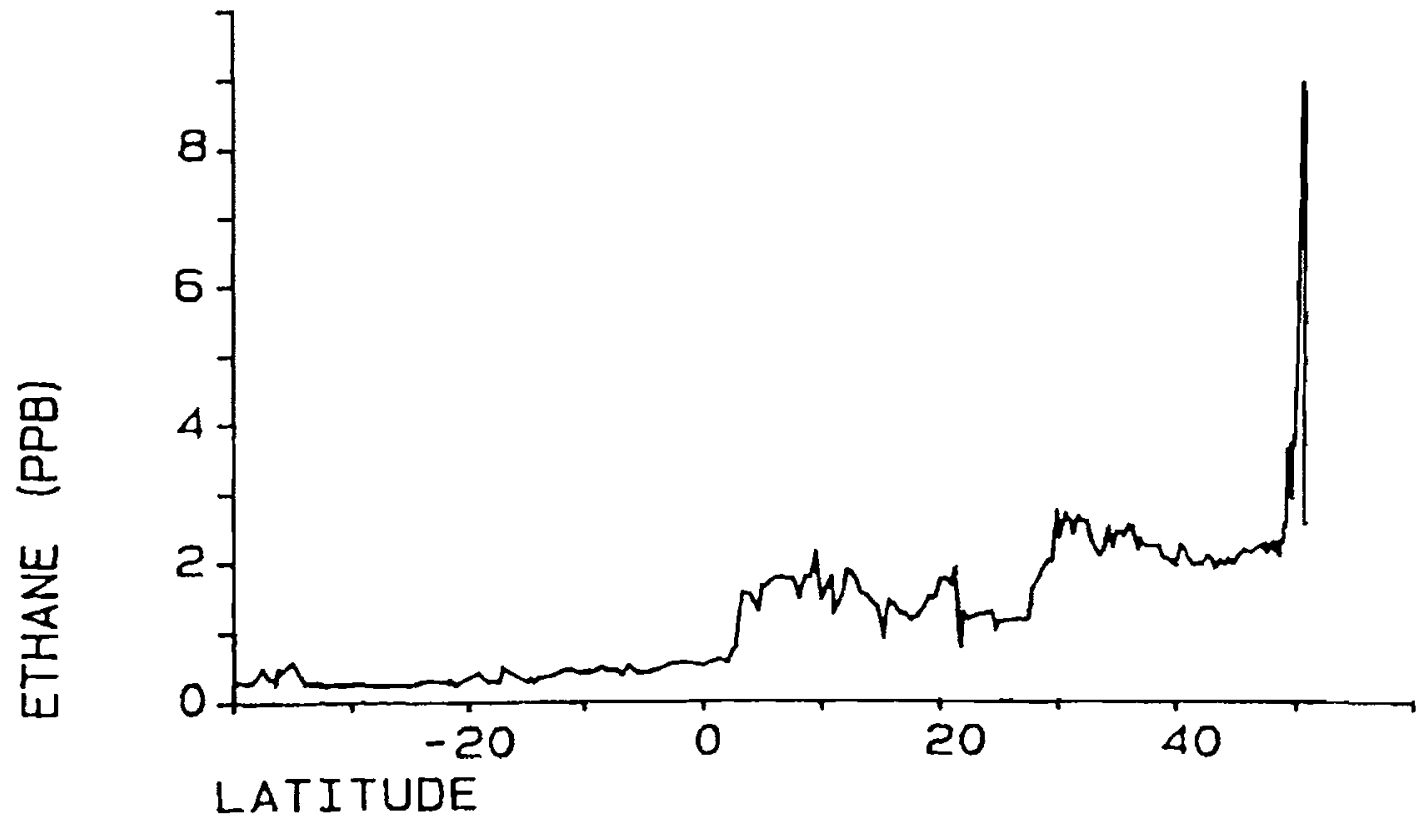

Figure 6 Latitudinal dependence of the atmospheric ethane mixing ratio.

to measure organic trace species in the non urban troposphere. Some of the results are shown here to demonstrate the usefulness of such techniques.

In 1987 we measured light organic trace gases during a ship cruise with F.S. Polarstern from Patagonia $\left(40^{\prime} \mathrm{S}\right)$ to Bremerhaven $\left(50^{\circ} \mathrm{N}\right)$. During this cruise of nearly 4 weeks duration we made more than 200 measurements with a frequency of three hours per measurement.

Figure 6 shows the atmospheric ethane mixing ratio as a function of latitude. The data not only show the increase in the ethane mixing between the southernand the northern hemisphere and the elevated levels of ethane around $50^{\circ} \mathrm{N}$, when the ship approached Europe. but also a number of minor features which can be ascribed to differences in the origin of the sampled air masses are clearly visible.

Examples for the variability of organic atmospheric trace consituents with time at a given place are shown in Figures 7 and 8 . These measurements were made during a 5 week campaign in a semirural area near Jülich (Federal Republic of Germany). Figure 7 demonstrates the change of the mixing ratios of ethene, acetylene and ethane within $24 \mathrm{~h}$ (one measurement every $3 \mathrm{~h}$ ). It can be seen, that the ethene mixing ratios change by a factor of more than 10 within a few hours. Acetylene shows a somewhat similar behaviour, but the changes are much less pronounced. The lowest variations are observed for ethane which has a rather long atmospheric residence time (global average about 2 month). ${ }^{11}$ In Figure 8 the ethene, ethane and benzene mixing ratios observed during a period of several days with a frequency of one sample every $3 \mathrm{~h}$ are shown. Again we can see a considerable short time variation of all three compounds. The three compounds show often quite similar structures in these time series measurements. The maxima and minima on the 14 and 15 May in general coincide for all three substances. Both benzene and ethene show very similar structures between the 9 and 13 May. although with differences in the magnitude of the individual "events". All three species show an increase at the end of the measurement series. In general, ethene 


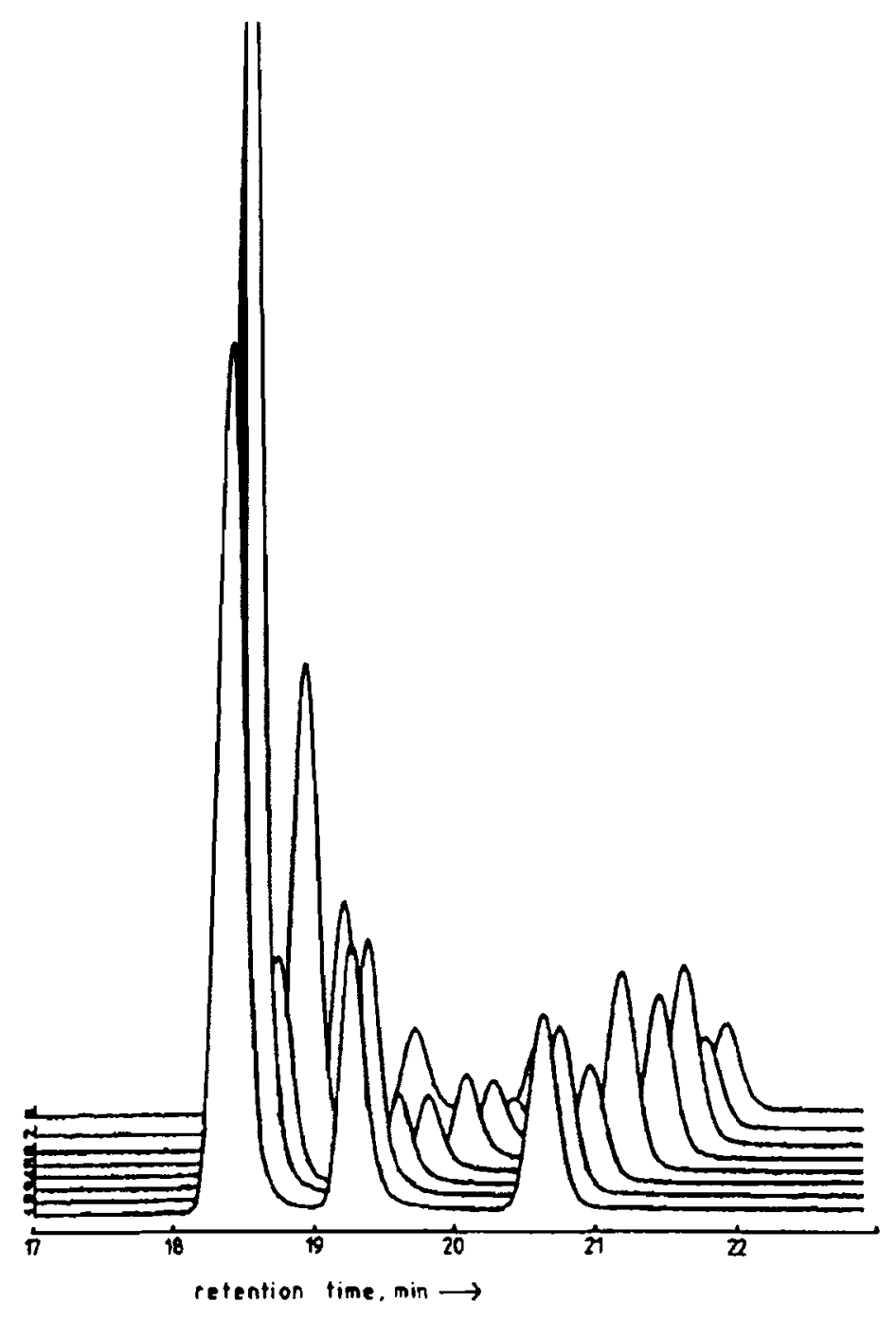

Figure 7 Chromatograms demonstrating the variation of $C_{2}$ hydrocarbons during a $24 \mathrm{~h}$ period (12 May 1988). First peak: ethene, second peak: acetylene, last peak ethane. Full scale corresponds to a mixing ratio of $15 \mathrm{ppb}$. measuring interval $3 \mathrm{~h}$.

and benzene show a very similar pattern. Several of the short term maxima and minima found for ethene and benzene are also observed for ethane, but often with a different amplitude. Also the average trend for ethane shows an increase between the 13 and 17. This change is less pronounced for benzene which has a shorter atmospheric residence time and extremely small for ethene, the shortest lived of these three hydrocarbons. Evidently, some of the changes are occurring on timescales of a few days, other within less than half a day.

These often occurring rather rapid changes in the mixing ratios of atmospheric organic trace constituents can only be followed if frequent measurements are made. This is especially important for very reactive species such as the alkenes. Due to the high photochemical reactivity such compounds do not only contribute significantly to important atmospheric reaction cycles, but also show considerable short term fluctuations even outside urban areas. 

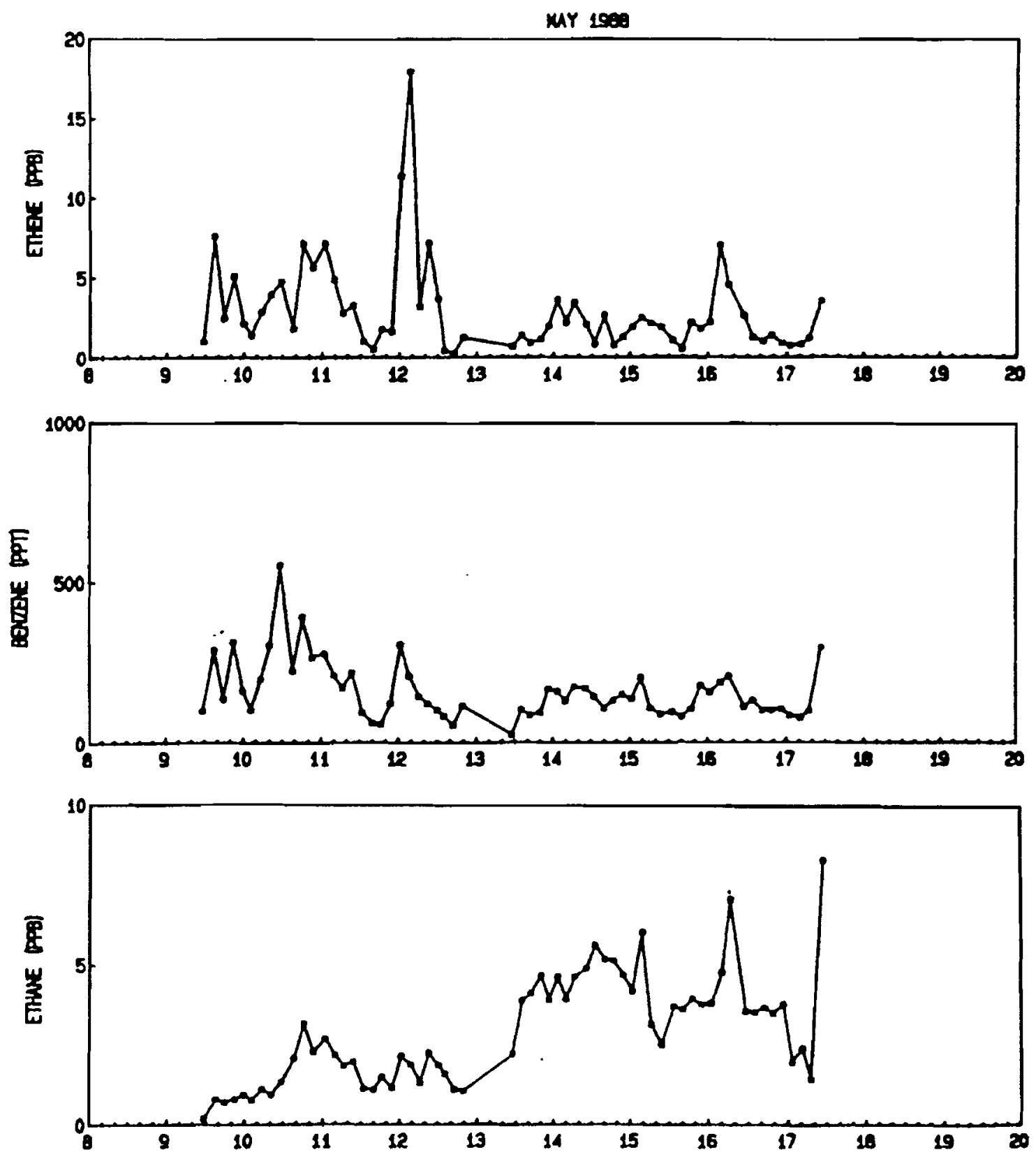

Figure 8 Time series measurements of ethane, ethene and benzene. 


\section{CONCLUSIONS}

Automated methods combining a cryogenic preconcentration step with gaschromatographic separation and detection are suitable for measurements of organic trace constituents in the non urban atmosphere. Lower limit of detection, reproducibility and linear range are in general sufficient to measure most of the relevant organic trace species even in remote areas. Sufficiently high measuring frequencies are important to understand the impact of organic trace gases on the chemistry of the atmosphere and the factors governing their atmospheric abundance.

\section{Acknowledgement}

This work was supported financially by the Bundesminister für Forschung und Technologie of the Federal Republic of Germany.

\section{References}

1. P. Matusca, M. Koval and W. Seiler, J. H RCC and CC 9, 577 (1986).

2. J. Rudolph. F. J. Johnen and A. Khedim, Int. J. Environ. Anal. Chem. 27, 97 (1986).

3. K. J. W. Tille, M. Savelsberg and K. Bächmann, Atm. Entiron. 19, 1751 (1985).

4. H. Knoeppel, B. Versino, H. Schlitt, A. Peil, H. Schauenburg and H. Vissers, Proc. of the First European Symposium on the Physico-Chemical Behaviour of Atmospheric Pollutants (B. Versino and H. Ott, eds) Ispra, 16-18 October 1979 (Ispra 1980) pp. 25-40.

5. R. Eichmann, P. Neuling, G. Ketseridis, J. Hahn, R. Jaenicke and C. Junge, Aim. Environ. 13, 587 (1979).

6. R. Eichmann, G. Ketseridis, G. Schebeske, R. Jainicke, J. Hahn, P. Warneck and C. Junge, Atm. Environ. 14, 695 (1980).

7. J. Rudolph and C. Jebsen, Int. J. Environ. Anal. Chem. 13, 129 (1983).

8. W. Nutamagul and D. R. Cronn, J. Atm. Chem. 2, 416 (1985).

9. J. Rudolph and A. Khedim, Int. J. Environ. Anal. Chem. 20, 265 (985).

10. R. A. Rasmussen, H. H. Westberg and M. Holdren, J. Chromatographic Sci. 12, 80 (1974).

11. J. Rudolph and D. H. Ehhalt, J. Geophy's. Res. 86, 11959 (1981). 